\title{
Produção científica sobre saúde penitenciária na enfermagem brasileira
}

Scientific production on penitentiary health in Brazilian nursing

Producción científica en salud penitenciaria en enfermería brasileña

\section{Mayara Lima Barbosa ${ }^{I}$, Alcides Viana de Lima Neto"I Cecília Olívia Paraguai de Oliveira Saraiva ${ }^{\mathrm{III}}$, Manacés dos Santos Bezerril ${ }^{\mathrm{IV}}$, Gabriela Maria Cavalcanti Costa ${ }^{\mathrm{v}}$, Viviane Euzébia Pereira Santos ${ }^{\mathrm{VI}}$}

\begin{abstract}
Resumo: Objetivo: caracterizar os estudos publicados no Centro De Estudos e Pesquisa em Enfermagem que abordam a temática saúde penitenciária. Método: trata-se de uma pesquisa bibliográfica, cuja busca foi realizada no banco de dados do CEPEn, nos catálogos disponíveis eletronicamente - correspondentes ao Volume XIX (2001) ao XXXIV (2016). Resultados: a análise foi realizada nos 9514 títulos constantes no banco de dados, entre os quais nove $(0,095 \%)$ das dissertações e teses foram desenvolvidas sobre o tema e compõem a amostra final. Destes, apenas um estudo relacionou enfermagem e saúde penitenciária e três destacaram implicações para a profissão. Conclusão: depreende-se que fomentar a discussão sobre o tema representa um avanço e é essencial para suprir as lacunas verificadas na prestação da assistência à saúde nesse cenário.

Descritores: Enfermagem; Prisões; Educação de Pós-Graduação em Enfermagem; Pesquisa em Enfermagem
\end{abstract}

\begin{abstract}
Objective: To characterize the studies published in the Center for Studies and Research in Nursing that address the theme penitentiary health. Method: This is a bibliographic search, which was searched in the CEPEn database, in the catalogs available electronically - corresponding to Volume XIX (2001) to XXXIV (2016). Results: The analysis was performed on the 9514 titles in the
\end{abstract}

\footnotetext{
${ }^{I}$ Enfermeira, Doutora em Enfermagem, Unifacisa, Campina Grande, Paraíba, Brasil. E-mail: mayaralimabarbosa@gmail.com, ORCID: https://orcid.org/0000-0002-8063-7903

II Enfermeiro, Mestre em Enfermagem, UFRN, Natal, Rio Grande do Norte, Brasil. E-mail: alcides.vln@gmail.com, ORCID: https://orcid.org/0000-0001-6191-9465

III Enfermeira, Doutora em Enfermagem, UFRN, Natal, Rio Grande do Norte, Brasil. E-mail: cecilia_olivia@yahoo.com.br, ORCID: https://orcid.org/0000-0003-4225-5194

IV Enfermeiro, Mestre em Enfermagem, UFRN, Natal, Rio Grande do Norte, Brasil. E-mail: manacesbezerril@hotmail.com, ORCID: https://orcid.org/0000-0002-9003-2334

V Enfermeira, Doutora em Enfermagem, UEPB, Campina Grande, Paraíba, Brasil. E-mail: gabrielamccost@gmail.com, ORCID: https://orcid.org/0000-0003-4283-8082

VI Enfermeira, Doutora em Enfermagem, UFRN, Natal, Rio Grande do Norte, Brasil. E-mail: vivianeepsantos@gmail.com, ORCID: https://orcid.org/0000-0001-8140-8320
} 
database, among which nine (0.095\%) dissertations and theses were developed on the subject and make up the final sample. Of these, only one study related nursing and penitentiary health and three highlighted implications for the profession. Conclusion: It is clear that fostering discussion on the topic represents a breakthrough and is essential to fill the gaps in health care delivery in this scenario. Descriptors: Nursing; Prisons; Nursing Graduate Education; Nursing Research

Resumen: Objetivo: caracterizar los estudios publicados en el Centro de Estudios e Investigación en Enfermería que abordan el tema de la salud penitenciaria. Método: Esta es una búsqueda bibliográfica, que se buscó en la base de datos CEPEn, en los catálogos disponibles electrónicamente, correspondientes al Volumen XIX (2001) a XXXIV (2016). Resultados: El análisis se realizó en los 9514 títulos de la base de datos, entre los cuales se desarrollaron nueve tesis (0.095\%) y tesis sobre el tema y constituyen la muestra final. De estos, solo un estudio se relacionó con la enfermería y la salud penitenciaria y tres destacaron las implicaciones para la profesión. Conclusión: está claro que fomentar la discusión sobre el tema representa un gran avance y es esencial para llenar los vacíos en la prestación de atención médica en este escenario.

Descriptores: Enfermería; Prisiones Educación de Posgrado en Enfermería; Investigación de enfermería

\section{Introdução}

A assistência a saúde é um direito garantido às pessoas privadas de liberdade no Brasil desde a Lei de Execuções Penais (LEP) no 7.210, de 11 de julho de $1984 .{ }^{1}$ No entanto, existiam lacunas que tornavam as ações de saúde fragmentadas, desarticuladas e pouco eficazes. Em virtude desse cenário preocupante e para reassumir o compromisso do Sistema Único de Saúde (SUS), no que se refere a atenção universal e equânime para toda a população brasileira, ${ }^{2}$ foi instituído pela Portaria Interministerial no 1777 , de 09 de setembro de 2003, o Plano Nacional de Saúde do Sistema Penitenciário (PNSSP). ${ }^{3}$

Após dez anos de publicação do PNSSP foi realizada uma grande avaliação e nesta percebeu-se que o plano já era ineficiente frente as necessidades de saúde da população privada de liberdade no país. Ante esta realidade foi criada a Política Nacional de Atenção Integral à Saúde das Pessoas Privadas de Liberdade no Sistema Prisional (PNAISP), instituída por meio da Portaria Interministerial № 1 , de 2 de janeiro de 2014. A PNAISP é regida pelos princípios de respeito aos direitos humanos 
e à justiça social, prevê a promoção de um ambiente humanizado, a integralidade da atenção à saúde da Pessoa Privada de Liberdade (PPL), a equidade e o reconhecimento da singularidade de cada indivíduo. ${ }^{4}$

A inserção de profissionais de saúde no âmbito penitenciário ocorreu desde a publicação da PNSSP e continuou após a instauração da PNAISP. De acordo com a política, o acesso e a atenção à saúde são desenvolvidos pelas Equipes de Atenção Básica no Sistema Prisional (EABP). Estas são formadas por um médico, um enfermeiro, um técnico de enfermagem ou auxiliar de enfermagem, um cirurgião dentista e um técnico ou auxiliar de saúde bucal, podendo ser acrescido um profissional de nível superior (fisioterapia, psicologia, assistência social, farmácia, terapia ocupacional, nutrição ou enfermagem) e/ou uma equipe de saúde mental, se necessário, considerando os critérios estabelecidos. ${ }^{4}$

É indispensável que os profissionais da EABP realizem suas práticas assistenciais em conformidade com aquelas ofertadas na atenção primária à saúde. Assim, deve ser garantido o fornecimento de atenção integral à saúde, a partir de ações que visam o tratamento e a reabilitação de doenças e agravos mais prevalentes, ações de prevenção de doenças e atividades de promoção da saúde, com a participação transversal da rede de cuidado. ${ }^{4}$

Diante da inserção dos profissionais de saúde no sistema penitenciário, as discussões sobre o tema ganharam espaço nos centros de formação. Em decorrência, apreende-se a necessidade de reconhecer como as pós-graduações de enfermagem têm pesquisado acerca da saúde penitenciária, ao considerar a importância da enfermagem no trabalho multidisciplinar desenvolvido no interior dos presídios brasileiros e o crescimento da área da Enfermagem Forense no país. 
Produção científica sobre saúde penitenciária na enfermagem brasileira | 4

Dessa forma, este estudo objetivou caracterizar os estudos publicados no Centro de Estudos e Pesquisa em Enfermagem que abordam a temática saúde penitenciária.

\section{Método}

Pesquisa bibliográfica, cuja busca foi realizada no Banco de dados do Centro de Estudos e Pesquisas em Enfermagem (CEPEn) da Associação Brasileira de Enfermagem (ABEn), em dezembro/2016, com atualização em setembro/2019, em todos os catálogos disponíveis eletronicamente que correspondem do Volume XIX (2001) ao XXXIV (2016).

A busca das produções de interesse presentes no CEPEN ocorreu por meio da utilização das seguintes palavras-chave: "saúde penitenciária”, “enfermagem forense”, “sistema prisional”, “sistema judicial” e "apenado”, com a utilização do operador boleano “OR”. O critério de inclusão foi: dissertações e teses produzidas por enfermeiros, componentes do CEPEn da ABEn e que versam sobre o tema saúde penitenciária.

Foram selecionados nove resumos de dissertações e teses sobre a temática em questão. As teses e dissertações dos resumos eleitos foram lidas na íntegra e analisadas quanto ao nível acadêmico da produção (mestrado ou doutorado), à instituição de ensino superior (IES) a que pertence o trabalho, ao ano de publicação, à região do país (Norte, Sul, Sudeste, Centro-oeste ou Nordeste), à unidade federativa brasileira que se encontra a IES, à área temática (Ensino, Pesquisa, Prática Assistencial, Gestão), ao objeto, objetivo e tipo do estudo, à abordagem metodológica (Qualitativa, quantitativa, mista) e às implicações e recomendações finais do trabalho. 


\section{Resultados}

A tabela 1 demonstra a pequena quantidade de estudos que foram desenvolvidos sobre saúde penitenciaria na pós-graduação em enfermagem (0,095\%). Há preponderância de estudos sobre o tema nos cursos de mestrado (88,9\%), em detrimento de cursos de doutorado (11,1\%). Em relação às instituições em que foram realizados, duas universidades, Universidade Federal de Santa Catarina (UFSC) e Universidade Federal da Paraíba (UFPB) se destacaram, com dois estudos cada uma. O ano de $2010(44,4 \%)$ apresentou-se com maior número de trabalhos realizados sobre saúde penitenciária. A região Nordeste se evidencia como maior produtora de estudos sobre a temática $(44,4 \%)$. Grande parte das dissertações e tese trata de pesquisas assistenciais (66,6\%), do tipo descritiva $(88,8 \%)$ e com abordagem metodológica qualitativa $(88,8 \%)$.

Tabela 1. Caracterização dos estudos quanto a nível acadêmico, Instituição de Ensino Superior, ano de publicação, região Unidade Federativa, área temática, tipo de estudo e abordagem. Brasil, 2019.

\begin{tabular}{lll}
\hline VARIÁVEL & N & $\mathbf{\%}$ \\
Nível Acadêmico & & \\
$\quad$ Doutorado & 1 & 11,1 \\
$\quad$ Mestrado & 8 & 88,9 \\
Instituição de Ensino Superior & & \\
$\quad$ Universidade Federal de Santa Catarina & 2 & 22,2 \\
$\quad$ Universidade Federal da Paraíba & 2 & 22,2 \\
$\quad$ Universidade Federal Bahia & 1 & 11,1 \\
Universidade Federal do Rio Grande do Norte & 1 & 11,1 \\
Universidade Federal de Goiás & 1 & 11,1 \\
Universidade Federal do Pará & 1 & 11,1 \\
Universidade Federal do Rio Grande & 1 & 11,1 \\
Ano de defesa do trabalho & & \\
2000 & 1 & 11,1 \\
2005 & 1 & 11,1 \\
2006 & 1 & 11,1 \\
2010 & 4 & 44,4 \\
2012 & 2 & 22,2 \\
Região da IES & & \\
Nordeste & & 44,4 \\
Sul & 4 & 33,3 \\
Centro-Oeste & 3 & 11,1 \\
\hline & 1 &
\end{tabular}


Produção científica sobre saúde penitenciária na enfermagem brasileira | 6

\begin{tabular}{lcc}
\hline Norte & 1 & 11,1 \\
$\begin{array}{l}\text { Área temática } \\
\text { Prática assistencial }\end{array}$ & 6 & 66,6 \\
$\quad$ Pesquisa & 3 & 33,4 \\
Tipo de estudo & & \\
$\quad$ Descritivo & 8 & 88,8 \\
$\quad$ Não informado & 1 & 11,2 \\
Abordagem metodológica & & \\
$\quad$ Qualitativa & 8 & 88,8 \\
$\quad$ Não informada & 1 & 11,2 \\
\hline
\end{tabular}

O Quadro 1 informa a caracterização das teses e dissertações quanto ao título, ao objeto, ao objetivo, às implicações e recomendações finais. Observa-se que apenas uma publicação apresenta objetivo direcionado para a enfermagem. O foco em avaliar as necessidades de saúde surgiu em duas pesquisas, uma voltada para a saúde em geral e outra para a saúde mental. A gestação, o parto e o puerpério foram objetivos de duas investigações. As doenças infectocontagiosas apresentaram-se como objeto mais recorrente nas produções da pós-graduação em enfermagem, com a realização de três estudos, um direcionado para HIV e outros dois para a Tuberculose.

No Quadro 1 também é possível observar que entre as implicações e recomendações finais, apenas três estudos fazem referência a enfermagem (1, 2 e 3), relacionada a contribuição para a assistência de enfermagem, ao fomento da discussão sobre a enfermagem na atenção à saúde no sistema prisional e para a formação de Enfermeira(o)s. 
7l Barbosa ML, Neto AVL, Saraiva COPO, Bezerril MS, Costa GMC, Santos VEP

\begin{tabular}{|c|c|c|c|c|}
\hline No & & & Objetivo & Implicações e recomendações finais \\
\hline 1 & $\begin{array}{l}\text { Enfermagem atrás das } \\
\text { grades: a saúde dos } \\
\text { presidiários }\end{array}$ & $\begin{array}{l}\text { Enfermagem atrás } \\
\text { das grades: a saúde } \\
\text { dos presidiários }\end{array}$ & $\begin{array}{l}\text { Apurar as necessidades de saúde dos } \\
\text { encarcerados e os problemas que agravam a } \\
\text { saúde na prisão; e construir protocolos de } \\
\text { enfermagem para a assistência aos encarcerados }\end{array}$ & $\begin{array}{l}\text { Pretende-se contribuir para a enfermagem na } \\
\text { assistência a presidiários ou familiares e a pessoas em } \\
\text { situações especiais }\end{array}$ \\
\hline 2 & $\begin{array}{l}\text { Mulheres presidiárias: } \\
\text { sobreviventes de um } \\
\text { mundo de sofrimento, } \\
\text { desassistência e privações }\end{array}$ & $\begin{array}{l}\text { Sistema carcerário } \\
\text { em nosso país } \\
\text { atualmente refere } \\
\text { ao direito à saúde }\end{array}$ & $\begin{array}{l}\text { Identificar as principais necessidades em saúde } \\
\text { mental de mulheres presidiárias; investigar a } \\
\text { presença de obstáculos que impedem o } \\
\text { atendimento às necessidades em saúde mental e } \\
\text { apontar estratégias de enfrentamento das } \\
\text { dificuldades tendo em vista a atenção à saúde } \\
\text { mental }\end{array}$ & $\begin{array}{l}\text { A inserção da enfermagem na discussão da atenção à } \\
\text { saúde no sistema prisional é algo novo que requer } \\
\text { continuidade, através de novas pesquisas científicas que } \\
\text { busquem contribuir com a promoção e prevenção da } \\
\text { saúde, tendo em vista as lacunas existentes na literatura } \\
\text { sobre o processo saúde-doença no cárcere }\end{array}$ \\
\hline 3 & $\begin{array}{l}\text { Impactos do } \\
\text { envolvimento } \\
\text { mulheres presidiárias } \\
\text { com o fenômeno das } \\
\text { drogas. }\end{array}$ & $\begin{array}{l}\text { Envolvimentos de } \\
\text { mulheres que } \\
\text { cumprem pena em } \\
\text { regime prisional } \\
\text { com as drogas }\end{array}$ & $\begin{array}{l}\text { Descrever os envolvimentos de mulheres que } \\
\text { cumprem pena sob regime prisional com o } \\
\text { fenômeno das drogas e identificar os impactos } \\
\text { decorrentes desses envolvimentos para as suas } \\
\text { vidas. }\end{array}$ & $\begin{array}{l}\text { Evidenciam desigualdades de gênero no tocante aos } \\
\text { envolvimentos das mulheres com as drogas e as suas } \\
\text { repercussões assinalando a urgência de investigações } \\
\text { sobre a temática. Ademais, aponta a necessidade de } \\
\text { inclusão e/ou ampliação da abordagem dessa } \\
\text { problemática na formação de Enfermeira(o)s. }\end{array}$ \\
\hline 4 & $\begin{array}{l}\text { Vivência de mulheres em } \\
\text { situação de cárcere } \\
\text { penitenciário durante o } \\
\text { período gestacional. }\end{array}$ & $\begin{array}{l}\text { Mulheres que } \\
\text { vivem a situação de } \\
\text { gestação em } \\
\text { cárcere } \\
\text { penitenciário }\end{array}$ & $\begin{array}{l}\text { Identificar as características sociodemográficas } \\
\text { e obstétricas de mulheres em situação de cárcere } \\
\text { penitenciário que vivenciaram a gestação no } \\
\text { Complexo Penal e descrever a vivência dessas } \\
\text { mulheres durante o período gestacional. }\end{array}$ & $\begin{array}{l}\text { Espera-se que o estudo possa dar visibilidade ao tema } \\
\text { pouco discutido na literatura e contribuir para a } \\
\text { construção de políticas públicas específicas para tal } \\
\text { realidade, como forma de minimizar os efeitos do } \\
\text { encarceramento durante o período gestacional. }\end{array}$ \\
\hline 5 & $\begin{array}{l}\text { Puerpério em ambiente } \\
\text { prisional: vivência de } \\
\text { mulheres }\end{array}$ & $\begin{array}{l}\text { Puerpério em } \\
\text { ambiente prisional }\end{array}$ & $\begin{array}{l}\text { Compreender como as detentas vivenciam o } \\
\text { puerpério em ambiente prisional }\end{array}$ & \\
\hline 6 & $\begin{array}{l}\text { Rastreamento sorológico } \\
\text { e epidemiologia da } \\
\text { infecção pelo vírus da }\end{array}$ & $\begin{array}{l}\text { Rastreamento } \\
\text { sorológico } \\
\text { epidemiologia }\end{array}$ & $\begin{array}{l}\text { Investigar o perfil soroepidemiológico da } \\
\text { infecção pelo vírus da Hepatite } B(\mathrm{HBV}) \\
\text { reeducandas da Regional Metropolitana de }\end{array}$ & $\begin{array}{l}\text { Os resultados evidenciam a necessidade de ações de } \\
\text { prevenção da Hepatite } B \text { no ambiente carcerário, } \\
\text { incluindo educação em saúde evacinação contra }\end{array}$ \\
\hline
\end{tabular}

Rev. Enferm. UFSM, Santa Maria, v. 9, e70, p. 1-15, 2019 
Produção científica sobre saúde penitenciária na enfermagem brasileira $\mid 8$

\begin{tabular}{|c|c|c|c|c|}
\hline & $\begin{array}{llr}\text { Hepatite } & \text { B } & \text { em } \\
\text { reeducandas } & \text { do } \\
\text { Complexo } & \text { Prisional da } \\
\text { Regional } & \text { Metropolitana } \\
\text { de Goiás } & \end{array}$ & $\begin{array}{l}\text { infecção pelo vírus } \\
\text { da Hepatite B }\end{array}$ & Goiás & Hepatite B. \\
\hline 7 & 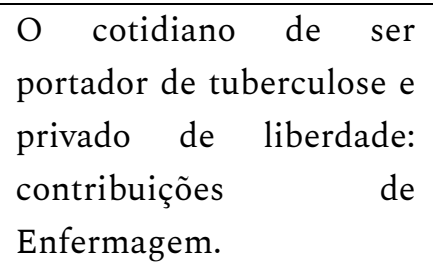 & $\begin{array}{lr}\text { O cotidiano de } & \text { ser } \\
\text { portador } & \text { de } \\
\text { tuberculose } & \text { e } \\
\text { privado } & \text { de } \\
\text { liberdade } & \end{array}$ & $\begin{array}{l}\text { Compreender o sentido que funda o cotidiano } \\
\text { de ser portador de tuberculose e privado de } \\
\text { liberdade. }\end{array}$ & $\begin{array}{l}\text { É necessário melhorar as condições de diagnóstico e } \\
\text { tratamento no ambiente prisional; discussões acerca do } \\
\text { tema devem ser ampliadas. }\end{array}$ \\
\hline 8 & $\begin{array}{l}\text { O discurso de pessoas } \\
\text { privadas de liberdade } \\
\text { com a tuberculose à luz } \\
\text { da história oral da vida. }\end{array}$ & $\begin{array}{lr}\text { Ações } & \text { de } \\
\text { da controle } & \text { Tuberculose } \\
\text { voltadas às } & \text { pessoas } \\
\text { privadas } & \text { de } \\
\text { liberdade } & \end{array}$ & $\begin{array}{l}\text { Analisar as ações de controle da tuberculose } \\
\text { voltadas às pessoas privadas de liberdade, } \\
\text { segundo as determinações do Plano Nacional de } \\
\text { Saúde no Sistema Penitenciário das unidades } \\
\text { penitenciárias masculinas do município de João } \\
\text { Pessoa, PB. }\end{array}$ & $\begin{array}{l}\text { A fim de superar as dificuldades quanto ao acesso à } \\
\text { saúde dos apenados e ao controle eficaz da tuberculose } \\
\text { entre essa população, necessita-se do empreendimento } \\
\text { de esforços, por parte do Estado, da gestão do presídio e } \\
\text { dos profissionais de saúde, na execução de ações em } \\
\text { saúde coesas com as políticas de saúde voltadas a esta } \\
\text { população e efetivação das Leis de Execuções Penais. }\end{array}$ \\
\hline 9 & $\begin{array}{l}\text { O processo de trabalho da } \\
\text { equipe de saúde com } \\
\text { pacientes apenados: um } \\
\text { estudo sobre metáforas e } \\
\text { estigma }\end{array}$ & $\begin{array}{lr}\text { Processo } & \text { de } \\
\text { trabalho da } & \text { equipe } \\
\text { de saúde } & \\
\text { com } & \text { clientes } \\
\text { apenados } & \text { no } \\
\text { interior de } & \text { uma } \\
\text { instituição } & \\
\text { hospitalar } & \end{array}$ & $\begin{array}{l}\text { Identificar como se desenvolve o processo de } \\
\text { trabalho da equipe de saúde em nível hospitalar } \\
\text { com o cliente apenado; e identificar a presença } \\
\text { de elementos que evidenciam uma relação } \\
\text { metafórica e estigmatizante com o cliente } \\
\text { detento }\end{array}$ & $\begin{array}{l}\text { A identificação da condição de seres humanos se faz } \\
\text { presente durante o processo de trabalho, possibilitando } \\
\text { tanto o afastamento do trabalhador do objeto de } \\
\text { trabalho (metáforas negativas geradas a partir do } \\
\text { estigma) quanto a aproximação dos mesmos (metáforas } \\
\text { positivas) }\end{array}$ \\
\hline
\end{tabular}

Quadro 1. Caracterização dos estudos quanto título, objeto, objetivo e implicações e recomendações finais. 
9 | Barbosa ML, Lima Neto AV, Saraiva COPO, Bezerril MS, Costa GMC, Santos VEP

\section{Discussão}

No Brasil, os cursos de mestrado e doutorado em enfermagem tem suas origens nas regiões Sudeste e Sul do país, com ênfase para os Estados do Rio de Janeiro e São Paulo. A partir de 1972, houve a expansão da pós-graduação para as demais localidades e, assim, também decorreu o aumento da produção científica, ${ }^{5}$ cuja qualidade é reconhecida pela comunidade acadêmica, em virtude do trabalho empreendido pelos programas de pós-graduação e pelos editores dos periódicos brasileiros. $^{6}$

Entretanto, é essencial impulsionar a internacionalização daquilo que é produzido nacionalmente, a fim de difundir os estudos brasileiros. Para tanto, é fundamental formar mestres e doutores para a utilização de abordagens teóricometodológicas complexas. ${ }^{6}$ Conforme se verifica, ainda há predominância de estudos descritivos que foram produzidos pelos programas de pós-graduação.

Neste estudo, demonstrou-se a preocupação dos pesquisadores da região Nordeste com a produção do conhecimento na área de saúde penitenciária. Essa constatação corrobora com a responsabilidade e empenho das instituições formadoras para a construção de um projeto educacional e de pesquisa que estivesse alinhado ao avanço da ciência e, sobretudo, que respondesse as necessidades de saúde da população. ${ }^{5}$

Sabe-se que no sistema penitenciário brasileiro há alta incidência de doenças infectocontagiosas e crônicas. Esse cenário relaciona-se como as carências diversificadas presente nas prisões, que se apresentam na estrutura arquitetônicas defasada, na superlotação das celas, na alimentação restrita e na higiene precária. É apreendido, por tanto, que a população privada de liberdade não tem diversos direitos respeitados, incluindo o da saúde. ${ }^{7}$ 
Produção científica sobre saúde penitenciária na enfermagem brasileira| 10

Este cenário decorre do abandono do sistema penitenciário pelos governantes, evidenciado pela falta ou timidez das políticas públicas resolutivas na área e, por conseguinte, nos pequenos investimentos realizados, que resultaram no sucateamento das unidades prisionais em todo o país. ${ }^{8}$ Este dado somado ao perfil sócio-demográfico da população privada de liberdade que, em geral, advém de comunidades socialmente desfavorecidas, resulta no fomento das vulnerabilidades e das iniquidades verificadas entre as pessoas que habitam as cadeias nacionais. ${ }^{9}$

Nesse sentido, produzir conhecimento/pesquisas sobre saúde penitenciária pode promover a saúde e fomentar ações que visem à qualidade de vida de pessoas vulneráveis, além de colaborar para a responsabilidade social que a produção científica assume nos dias atuais. ${ }^{10} \mathrm{E}$ ainda, contribuir para a formação de enfermeiros com maiores níveis de qualificação, preparados para a atuação em diversos âmbitos e complexidades, no que se refere ao cuidado a saúde da pessoa e da coletividade. ${ }^{5}$

O número de estudos sobre saúde penitenciária mostra-se insipiente na literatura nacional, mesmo em face do crescente interesse da pós-graduação em enfermagem em pesquisar sobre o tema. Diversos fatores podem estar relacionados ao pequeno número de pesquisas realizadas no sistema penitenciário como a insipiente disseminação de informação sobre saúde penitenciária durante a formação profissional; ${ }^{11}$ a necessidade de conhecimento básico sobre o sistema administrativo das unidades prisionais; a seleção de abordagens metodológicas adequadas para o cenário, cujo eixo prioritário é a segurança; o estigma do pesquisador e o estabelecimento de redes de contato com o setor administrativo, de saúde, de segurança e com as PPL. ${ }^{12}$

Contudo, no Brasil, após a publicação do Plano Nacional de Saúde do Sistema Penitenciário (PNSSP); da inserção dos profissionais de saúde nas unidades prisionais 
11 | Barbosa ML, Lima Neto AV, Saraiva COPO, Bezerril MS, Costa GMC, Santos VEP

nacionais e da ampliação do direito à saúde por parte das PPL, houve maior estímulo à produção de pesquisas desenvolvidas na área da saúde. ${ }^{3}$

Destaca-se o número de pesquisas encontradas que utilizaram os métodos qualitativos para o alcance dos objetivos. Tal realidade mostra-se relacionada a particularidades dessa abordagem metodológica, como a possibilidade de explorar temas pouco conhecidos, elucidar questões norteadoras insuficientemente respondidas e apreender aspectos diretamente retratados pelos discursos dos atores envolvidos. ${ }^{13}$

Ênfase também foi dada a pesquisas assistenciais que se caracterizam por estudos que propiciam responder questões que emergem das práticas profissionais. Esse tipo de produção intelectual tem sido difundido e utilizado por pesquisadores enfermeiros, em virtude da possível redução da lacuna existente entre teoria e prática ao produzir inovações e melhorias in $10 c{ }^{14}$

Parte das pesquisas desenvolvidas na pós-graduação em enfermagem no Brasil objetivou avaliar as necessidades de saúde da população privada de liberdade e estas podem contribuir para a resolução dos problemas de saúde mais evidentes no cenário superlotado das unidades prisionais nacionais. Entre os agravos à saúde mais emergentes destaca-se a violência, o uso abusivo de drogas e os altos índices de doenças mentais e infectocontagiosas. ${ }^{12}$

Apenas um estudo direcionou seu objetivo para a enfermagem, que correspondeu a criação de protocolos de enfermagem para a assistência as PPL após a verificação das necessidades de saúde dessa população. Isto posto, é fundamental que a enfermagem produza ciência relevante, inovadora e que some ao conhecimento já existente.

A avaliação realizada em virtude dos 40 anos de pós-graduação em enfermagem, no Brasil, afirma a necessidade de desenvolver pesquisas cujos objetivos alcancem resultados expressivos para os serviços de saúde. Tal constatação é consonante com 
Produção científica sobre saúde penitenciária na enfermagem brasileira| 12 as necessidades da coletividade, contribuem para a consolidação do Sistema Único de Saúde e para a disciplina e profissão. ${ }^{5}$

Em relação as implicações dos estudos desenvolvidos, alguns autores destacaram a contribuição para as práticas assistenciais de enfermagem no âmbito prisional brasileiro. Pesquisas desse espectro contribuem para resolver problemas advindos das práticas e garantir a saúde integral das PPL, tendo em vista que o processo de trabalho da equipe de saúde penitenciária apresenta características específicas, relacionadas a periculosidade das pessoas atendidas ou pelos protocolos de segurança. ${ }^{15}$

Por fim, ainda foi destacada a necessidade de melhorar a estrutura das unidades de saúde no ambiente penitenciário. As más condições de trabalho dos profissionais de saúde nas penitenciárias interferem negativamente sobre a qualidade da assistência ofertada, ${ }^{16}$ aumentando a insatisfação profissional relacionada ao exercício laboral e a carga de trabalho dos profissionais. ${ }^{17}$

\section{Considerações finais}

O número de iniciativas de desenvolvimento de pesquisas sobre saúde penitenciária na pós-graduação em enfermagem brasileira ainda é pequena, conforme os resultados verificados. Os estudos publicados na base de dados que abordam a temática caracterizam-se por, em grande parte, terem sido resultado de cursos de mestrado, nas universidades federais de Santa Catarina e Paraíba, após o ano de 2010, decorrentes de abordagens qualitativas, descritivas e assistenciais.

Em relação ao objetivo, apenas um estudo fez menção a disciplina enfermagem, sendo que os demais buscaram avaliar as necessidades de saúde de maneira geral ou com ênfase para a saúde mental. Os demais temas versaram sobre gestação, o parto e o puerpério, uso de drogas, processo de trabalho e doenças infectocontagiosas. 
13 | Barbosa ML, Lima Neto AV, Saraiva COPO, Bezerril MS, Costa GMC, Santos VEP

As implicações dos estudos retrataram os ganhos para as práticas assistenciais da enfermagem, assim como a necessidade de realização de outras pesquisas sobre o tema, a possibilidade de subsídio de políticas públicas de saúde direcionados para as PPLs e ainda indicaram a necessidade de realização das ações de saúde executadas no âmbito prisional ou sua qualificação.

Apreende-se que a pós-graduação em enfermagem, desde a criação de políticas públicas de saúde específicas para a saúde penitenciária e a decorrente inserção de profissionais enfermeiros nas unidades prisionais, tem voltado sua atenção para a produção de estudos sobre o tema. Isso tem acontecido de maneira tímida, principalmente na região sudeste do país.

Contudo, fomentar a discussão sobre o tema saúde penitenciária representa um avanço e é essencial para suprir as lacunas verificadas na prestação da assistência à saúde da PPL, a fim de buscar a defesa da vida, da dignidade, da qualidade de vida e dos direitos inerentes a pessoa humana, independente de onde habitam.

As limitações desse estudo estão relacionadas à falta de padronização dos resumos que constam na base de dados selecionadas, o que pode interferir negativamente na inclusão das teses e dissertações neste estudo de revisão. Em tempo, sugere-se o fomento de pesquisas que associam saúde penitenciária e enfermagem, bem como a melhor estruturação dos resumos publicados no CEPEn.

\section{Referencias}

1. Ministério da Justiça (BR). Casa Civil. Subchefia para Assuntos Jurídicos Lei de Execução Penal, Lei no 7.210, de 11 de julho de 1984: institui a Lei de Execução Penal. Brasília: Poder Executivo. 1984.

2. Brasil. Constituição da República Federativa do Brasil. Brasília: Poder Executivo 1988. 
3. Ministério da Saúde (BR). Secretaria de Atenção à Saúde. Departamento de Ações Programáticas Estratégicas. Portaria Interministerial no 1777, de 09 de setembro de 2003: institui o Plano Nacional de Saúde no Sistema Penitenciário. Brasília: Ministério da Saúde; 2003.

4. Ministério da Saúde (BR). Secretaria de Atenção à Saúde. Departamento de Ações Programáticas Estratégicas. Portaria Interministerial № 1, de 2 de janeiro de 2014:instituiu a Política Nacional de Atenção Integral à Saúde das Pessoas Privadas de Liberdade no Sistema Prisional. Brasília: Ministério da Saúde; 2014.

5. Scochi CGS, Munari DB, Gelbcke FL, Ferreira MA. Desafios e estratégias dos programas de pós-graduaçãoem enfermagem para a difusão da produção científica emperiódicos internacionais. Esc Anna Nery. 2014;18(1):05-10

6. Scochi CGS et al. Pós-Graduação Stricto Sensu em Enfermagem no Brasil: avanços e perspectivas. Rev Bras Enferm. 2013;66(esp):80-9.

7. Soares Filho MM, Bueno PMMG. Demografia, vulnerabilidades e direito à saúde da população prisional brasileira. Ciênc. saúde coletiva. 2016; 21(7): 1999-2010.

8. Machado AEB, Souza APR, Souza MC. Sistema Penitenciário Brasileiro - Origem, Atualidade e Exemplos Funcionais. Rev Curso de Direito. 2013; 10(10): 201-212.

9. Delziovo CR et al, organizadores. Atenção à saúde da mulher privada de liberdade. Florianópolis (SC): Universidade Federal de Santa Catarina, 2015.

10. Sánchez A, Leal MC, Larouzé B. Realidade e desafios da saúde nas prisões. Ciênc. saúde coletiva. 2016; 21(7):1996.

11. Diniz RCM. A formação dos trabalhadores da saúde do Sistema Penitenciário cartografia dos saberes e práticas [tese]. Fortaleza (CE): Faculdade de Educação, Universidade Federal do Ceará; 2011.

12. Apa, ZL. et al. "Challenges and Strategies for Research in Prisons." Public health nursing. 2012; 29(5): 467-72.

13. Kerr LRFS, kendall C. A pesquisa qualitativa em saúde. Rev Rene. 2013; 14(6):1061-3.

14. Pivoto FL, Filho WDL, Santos SSC, Lunardi VL. Pesquisa convergente-assistencial: revisão integrativade produções científicas da enfermagem. Texto Contexto Enferm. 2013; 22(3): 843-9.

15. Barbosa ML, Celino SDM, Oliveira LV, Pedraza DF, Costa GMC. Atenção básica à saúde de apenados no sistema penitenciário: subsídios para a atuação da enfermagem. Esc. Anna Nery. 2014; 18(4): 586-592. 
16. Costa GMC, Barbosa ML, Celino SDM, Oliveira LV. Perfil demográfico e das condições de trabalho: a realidade das equipes de saúde implantadas em unidades prisionais. Rev. Bras. Pesq. Saúde. 2014; 16(4): 13-22.

17. Santos DA et al. Potencialidades e dificuldades nas práticas de acolhimento na rede de atenção básica conforme a Política Nacional de Humanização. Sau. \& Transf. Soc. 2016; 6 (2): 54.-69.

\section{Autor correspondente}

Mayara Lima Barbosa

E-mail: mayaralimabarbosa@gmail.com

Endereço: Rua João Albuquerque Santiago, 181, Catolé, Campina Grande, Paraíba, Brasil

CEP: $58410-532$

\section{Contribuições de Autoria}

\section{1 - Mayara Lima Barbosa}

Concepção e planejamento do projeto de pesquisa, obtenção ou análise e interpretação dos dados, redação e revisão crítica.

\section{2 - Alcides Viana de Lima Neto}

Obtenção ou análise e interpretação dos dados, redação e revisão crítica.

\section{3 - Cecília Olívia Paraguai de Oliveira Saraiva}

Obtenção ou análise e interpretação dos dados, redação e revisão crítica.

\section{4 - Manacés dos Santos Bezerril}

Obtenção ou análise e interpretação dos dados, redação e revisão crítica.

\section{5- Gabriela Maria Cavalcanti Costa}

Redação e revisão crítica.

\section{6 - Viviane Euzébia Pereira Santos}

Concepção e planejamento do projeto de pesquisa, obtenção ou análise e interpretação dos dados, redação e revisão crítica.

\section{Como citar este artigo}

Barbosa ML, Lima Neto AV, Saraiva COPO, Bezerril MS, Costa GMC, Santos VEP. Produção científica sobre saúde penitenciária na enfermagem brasileira. Rev. Enferm. UFSM. 2019 [Acesso em: Anos Mês Dia];vol9, e70: 1-15. DOI:https://doi.org/10.5902/2179769232885 